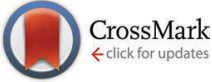

Cite this: Phys. Chem. Chem. Phys., 2017, 19, 7853

Received 4th October 2016, Accepted 24th February 2017

DOI: $10.1039 / c 6 c p 06793 j$

rsc.li/pccp

\title{
Imaging rotations and vibrations in polyatomic molecules with X-ray scattering
}

\author{
Andrés Moreno Carrascosa, Thomas Northey and Adam Kirrander*
}

\begin{abstract}
An approach for calculating elastic X-ray scattering from polyatomic molecules in specific electronic, vibrational, and rotational states is presented, and is used to consider the characterization of specific states in polyatomic molecules using elastic X-ray scattering. Instead of the standard independent atom model (IAM) method, the $X$-ray scattering is calculated directly from ab initio wavefunctions. The role of molecular symmetry and Friedel's law is examined, with the molecules $\mathrm{BF}_{3}, \mathrm{C}_{5} \mathrm{H}_{5}{ }^{-}, \mathrm{NF}_{3}$, and 1,3-cyclohexadiene used as specific examples. The contributions to the elastic $\mathrm{X}$-ray scattering from the electronic, vibrational, and rotational portions of the molecular wavefunction are examined in $\mathrm{CS}_{2}$. In particular, it is observed that the rotational states give rise to distinct signatures in the scattering signal.
\end{abstract}

\section{Introduction}

X-ray Free-Electron Lasers (XFELs) have higher intensity and shorter pulse durations than synchrotrons, and have been adopted for many experiments including X-ray spectroscopy, ${ }^{1,2}$ Coulomb explosion imaging, ${ }^{3}$ and ultrafast scattering. ${ }^{4,5}$ Elastic scattering of X-rays, in particular, provides valuable insight into molecular structure and function. An attractive feature of XFELs is that the high intensity of the radiation reduces the need for crystals to amplify the scattered signal, with important implications for e.g. the diffractive imaging of biomolecules. ${ }^{6-8}$ In the absence of a crystal, the Bragg peaks disappear and the scattering image becomes continuous. Furthermore, the short pulse duration of XFELs limits the effect of radiation damage on the scattering signal. $^{7,8}$

Gas-phase X-ray scattering ${ }^{9-13}$ is currently undergoing a revival. ${ }^{4,14-17}$ The main reason for this is that the high intensity of XFELs can compensate for small X-ray scattering cross sections, albeit gas-phase scattering is also possible at synchrotrons. ${ }^{18-21}$ An important aspect is that rapid development of alignment and orientation techniques ${ }^{22}$ and experimental methods to prepare molecules in specific quantum states ${ }^{23-27}$ makes it possible to generate highly anisotropic samples with a large fraction of identical molecules. As a consequence, the degree of thermal averaging in the data becomes small, invalidating the incoherent thermal averaging of the signal originally proposed by Debye. ${ }^{28}$ All these factors, i.e. continuous scattering, limited accumulation of radiation damage, non-thermal or

EaStCHEM, School of Chemistry, University of Edinburgh, David Brewster Road, EH9 3FJ Edinburgh, UK. E-mail: Adam.Kirrander@ed.ac.uk even state-selected samples will contribute to more complete and detailed data sets, that will require more sophisticated analysis.

Standard analysis of diffraction data relies on the independent atom model (IAM), which uses tabulated atomic form factors calculated for single atoms at the Hartree-Fock (HF) level ${ }^{29-31}$ to assemble an approximate representation of the scattering. In thermal samples, this is generally sufficient, ${ }^{32-34}$ although wellknown examples exist ${ }^{35,36}$ where the failure of IAM to account for changes in electron density due to e.g. chemical bonding renders IAM inadequate. Given the advances in electronic structure calculations, an interesting improvement on IAM is to calculate molecular scattering form factors directly from the molecular wavefunctions. Potentially this could provide the most flexible, general, and accurate approach to calculating X-ray scattering. ${ }^{37-41}$ Our recently developed AIXRD code ${ }^{42}$ does exactly this.

In the following, we consider the effect of rotational and vibrational motion on the scattering pattern of polyatomic molecules, and show that these can be incorporated into our AIXRD treatment. We explore the properties of state-specific scattering patterns from polyatomic molecules, and assess the impact of vibrational, rotational and electronic states on the scattering signal in $\mathrm{CS}_{2}$, an important molecule in gasphase spectroscopy and dynamics. ${ }^{43-48}$ We also examine the convergence of AIXRD calculations compared to IAM, with the molecules $\mathrm{NH}_{3}$ and $\mathrm{BF}_{3}$ used as specific examples, and the computational requirements with regards to the basis set used. The consequences of Friedel's law and molecular symmetry on the scattering patterns are also examined, with the molecules $\mathrm{BF}_{3}, \mathrm{C}_{5} \mathrm{H}_{5}{ }^{-}, \mathrm{NF}_{3}$, and 1,3-cyclohexadiene used as examples. 


\section{Theory}

\subsection{X-ray scattering}

For static X-ray scattering, the total differential cross section is given by Fermi's golden rule, ${ }^{49}$

$$
\frac{\mathrm{d} S}{\mathrm{~d} \Omega}=\left(\frac{\mathrm{d} \sigma}{\mathrm{d} \Omega}\right)_{\mathrm{Th}} \sum_{m}\left(\frac{\omega_{m}}{\omega_{0}}\right)\left|\left\langle\phi_{m}|\hat{L}| \phi_{n}\right\rangle\right|^{2},
$$

where $\phi_{m}$ and $\phi_{n}$ are the final and initial states, and correspondingly $\omega_{m}$ and $\omega_{0}$ are the angular frequencies of the scattered and incident X-rays. The pre-factor $(\mathrm{d} \sigma / \mathrm{d} \Omega)_{\mathrm{Th}}=\left(e^{2} / m_{e} c^{2}\right) K$ is the Thomson cross section of a free electron, with $m_{e}$ and $e$ the mass and charge of an electron, $c$ the velocity of light, and $K$ the polarization factor. Ignoring the weak interaction between the electromagnetic field and the nuclei, the scattering operator $\hat{L}$ is defined as,

$$
\hat{L}=\sum_{j=1}^{N_{\mathrm{el}}} \mathrm{e}^{l \mathbf{q r} \mathbf{r}_{j}}
$$

where the sum runs over the $N_{\mathrm{el}}$ electrons in the molecule, with $\mathbf{r}_{j}$ the electron coordinate and $\mathbf{q}=\mathbf{k}_{0}-\mathbf{k}$ the momentum transfer vector. In this article, we focus on the elastic scattering contribution as a means to characterise molecular states. The elastic scattering is proportional to the matrix element $L_{n n}$ in eqn (1), i.e.

$$
\tilde{I}_{n}(\mathbf{q})=\left|L_{n n}\right|^{2}=\left|\left\langle\phi_{n}|\hat{L}| \phi_{n}\right\rangle\right|^{2},
$$

which is also known as the structure factor, and which we denote as $\tilde{I}_{n}(\mathbf{q})$. For elastic scattering, the incident $\left(\mathbf{k}_{0}\right)$ and scattered (k) wave vectors have the same length, $|\mathbf{k}|=\left|\mathbf{k}_{0}\right|$.

\subsection{Scattering from molecular wavefunctions}

In order to calculate the structure factor $\tilde{I}_{n}(\mathbf{q})$ in eqn (3), we require a representation of the molecular state $\left|\phi_{n}\right\rangle$. Using the Born-Oppenheimer ansatz and assuming that the rotationalvibrational coupling is small, and that electronic states are sufficiently well separated that non-adiabatic couplings can be ignored, the state $n$ can be written as a direct product of rotational, vibrational and electronic wavefunctions,

$$
\left|\phi_{n}\right\rangle=\Psi_{J K M}^{\mathrm{rot}}(\Omega) \Psi_{\nu}^{\mathrm{vib}}(\overline{\mathbf{R}}) \Psi_{\alpha}^{\mathrm{elec}}(\overline{\mathbf{r}} ; \overline{\mathbf{R}}, \Omega)
$$

where the electronic wavefunction, $\Psi_{\alpha}^{\text {elec }}(\overline{\mathbf{r}} ; \overline{\mathbf{R}}, \Omega)$, depends parametrically on the nuclear coordinates $\overline{\mathbf{R}}$ and on the orientation of the molecular frame specified by the rotational Euler angles $\Omega=(\alpha, \beta, \gamma)$ (see Appendix). The rotational wavefunction $\Psi_{J K M}^{\mathrm{rot}}(\Omega)$ is characterized by the three rotational quantum numbers $J, K$, and $M$, and the vibrational wavefunction $\Psi_{\nu}^{\mathrm{vib}}(\overline{\mathbf{R}})$ by the full set of vibrational quantum numbers $\nu$. Since the scattering operator in eqn (2) acts on the electrons, it is convenient to first evaluate the scattering in terms of the form factor for the electronic wavefunction,

$$
f_{\alpha}^{0}(\mathbf{q} ; \overline{\mathbf{R}}, \Omega)=\left\langle\Psi_{\alpha}^{\text {elec }}|\hat{L}| \Psi_{\alpha}^{\text {elec }}\right\rangle .
$$

The form factor $f_{\alpha}^{0}(\mathbf{q} ; \overline{\mathbf{R}}, \Omega)$ can be calculated directly from the $a b$ initio electronic wavefunction..$^{39,42,50}$ For $a b$ initio wavefunctions constructed from Gaussian primitives, the calculation

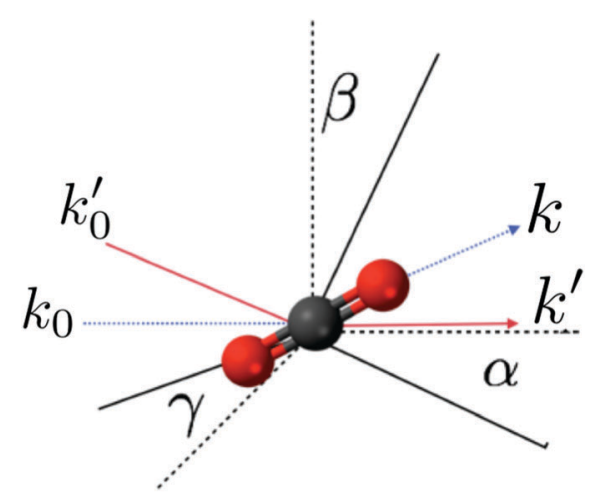

Fig. 1 Schematic illustration of how the rotation of the molecule through Euler angles $(\alpha, \beta, \gamma)$ can be substituted by the inverse rotation of the momentum transfer vector $\mathbf{q}=\mathbf{k}_{0}-\mathbf{k}$. The components $\mathbf{k}_{0}$ and $\mathbf{k}$ are transformed into $\mathbf{k}_{0}{ }^{\prime}$ and $\mathbf{k}^{\prime}$ by the inverse rotation matrix. In the figure the molecular and laboratory frames are rotated to show the equivalence of both rotations. The dashed lines represent the original axis and vectors, and the solid lines the final positions.

of $f_{\alpha}^{0}(\mathbf{q} ; \overline{\mathbf{R}}, \Omega)$ can be done semi-analytically, ${ }^{42}$ although numerical Fourier transforms of the electron density represented on a grid are also possible. ${ }^{42}$

The structure factor, $\tilde{I}_{n}(\mathbf{q})$, can thus be calculated as a convolution of $f_{\alpha}^{0}(\mathbf{q} ; \overline{\mathbf{R}}, \Omega)$ by the vibrational and rotational probability distributions,

$$
\tilde{I}_{\tilde{\alpha}}(\mathbf{q})=\left.\left.\left|\int\right| \Psi_{J K M}^{\mathrm{rot}}(\Omega)\right|^{2}\left|\Psi_{\nu}^{\mathrm{vib}}(\overline{\mathbf{R}})\right|^{2} f_{\alpha}^{0}(\mathbf{q} ; \overline{\mathbf{R}}, \Omega) \mathrm{d} \overline{\mathbf{R}} \mathrm{d} \Omega\right|^{2} .
$$

It is worth pointing out that the rotational averaging undertaken in eqn (6) is different from the isotropic and incoherent rotational averaging normally used for thermal samples, first derived by Debye, ${ }^{28}$ whereby $\left|f_{\alpha}^{0}(\mathbf{q} ; \overline{\mathbf{R}}, \Omega)\right|^{2}$ is uniformly integrated over all directions of $\mathbf{q}{ }^{51}$

The multiconfigurational electronic wavefunctions used to calculate the form factor in eqn (5) have been discussed at length in ref. 42 , but we consider here in some further detail the vibrational and rotational wavefunctions that enter eqn (6) since these were not accounted for in the previous treatment. The vibrational wavefunctions are represented as harmonic oscillators, with normal modes and associated frequencies obtained from the Hessian of the ab initio molecular wavefunction at optimised energy minima. Since only small values of the vibrational quantum numbers $\nu$ are considered here, anharmonic regions of the potential energy surface are avoided. In terms of the rotational wavefunctions, we do not include rotational coupling, and can therefore fully determine the wavefunctions from the rotational constants and associated quantum numbers. Further details on the rotational wave functions used are given in the Appendix.

One important point regarding the rotational convolution in eqn (6) relates to the fact that the form factor $f_{\alpha}^{0}(\mathbf{q} ; \overline{\mathbf{R}}, \Omega)$ depends on the orientation of the molecular frame in the laboratory (scattering) frame. When the electronic wavefunction rotates through the Euler angles $\Omega=(\alpha, \beta, \gamma)$, the wavefunction expansion coefficients change. The rotated coefficients can be determined 
by re-calculating the electronic wavefunction in the rotated molecular frame, but this is inefficient. A slightly better approach is to rotate the electronic wavefunction directly, but this requires that the coefficients for all Gaussian primitives with $l>0$ are transformed. Ultimately, the simplest and computationally most efficient approach is to rotate $\mathbf{q}$ rather than the molecule. This amounts to applying the inverse (i.e. transpose) of the rotation matrix to the vector $\mathbf{q}$ for each set of rotation angles $(\alpha, \beta, \gamma)$, while leaving the electronic wavefunction fixed in the original molecular frame used in the ab initio calculation. An attempt to illustrate the equivalence of these two scenarios, i.e. rotating the molecule versus rotating $\mathbf{q}$, is shown in Fig. 1.

\subsection{Effective electron density and IAM}

One of the main reasons why elastic X-ray scattering is such an important tool for structure determination ${ }^{32}$ is that the form factor corresponds to a Fourier transform of the electron density, $\rho_{\alpha}(\mathbf{r} ; \overline{\mathbf{R}}, \Omega)$,

$$
f_{\alpha}^{0}(\mathbf{q} ; \overline{\mathbf{R}}, \Omega)=\left\langle\Psi_{\alpha}^{\mathrm{elec}}|\hat{L}| \Psi_{\alpha}^{\mathrm{elec}}\right\rangle=\int \rho_{\alpha}(\mathbf{r} ; \overline{\mathbf{R}}, \Omega) \mathrm{e}^{l \mathbf{q r}} \mathrm{d} \mathbf{r}
$$

thus giving direct access to the "shape" of a molecule. In the independent atom model (IAM) ${ }^{32}$ the electron density is approximated by a sum of spherical single-atom densities, which yield the form factor as a sum of atomic form factors pre-fixed by a phase factor that depends on the relative positions of the atomic nuclei. The convenience of IAM stems from the fact that the atomic form factors have been calculated and tabulated for all future use, ${ }^{33}$ but the drawback is that distortions in the electron distribution, for instance due to molecular bonding or electronic excitations, are not included. This situation can to some extent be alleviated by the use of generalized form factors fitted to the distorted charge distributions for the bonded atoms ${ }^{33,35,36}$ for instance to describe hydrogen atoms in organic molecular crystals.

An interesting point is that the electron density observed in a gas-phase experiment corresponds to the total molecular wavefunction and hence the structure factor $\tilde{I}_{n}(\mathbf{q})$ in eqn (3) and (6). This structure factor is the Fourier transform of the total (effective) electron density,

$$
\rho_{n}^{\text {effective }}(\mathbf{r})=\int\left|\Psi_{J K M}^{\mathrm{rot}}(\Omega)\right|^{2}\left|\Psi_{\nu}^{\mathrm{vib}}(\overline{\mathbf{R}})\right|^{2} \rho_{\alpha}(\mathbf{r} ; \overline{\mathbf{R}}, \Omega) \mathrm{d} \overline{\mathbf{R}} \mathrm{d} \Omega
$$

rather than the molecular electron density $\rho_{\alpha}(\mathbf{r} ; \overline{\mathbf{R}}, \Omega)$ in eqn (7). This changes the meaning of the "shape" of the molecule, with the rotational and vibrational wavefunctions beginning to play an important role. For instance, a homonuclear diatomic in the ground rotational and vibrational state appears as a hollow spherical shell, while the same molecule in the first excited vibrational state appears as two spherical shells, one inside the other. This clearly differs from the picture of an ensemble of "ball-and-stick" diatomic molecules, each in a random orientation, leading to an incoherent thermal averaging of $\left|f_{\alpha}^{0}\right|^{28,51}$

\section{Computational}

The $a b$ initio electronic structure calculations have been carried out using Molpro. ${ }^{52}$ The wavefunctions for ground state molecules have been calculated using Hartree-Fock (HF) theory, except for the $\mathrm{CS}_{2}$ molecule, where multiconfigurational self-consistent field theory (CAS-SCF) with an active space of $(10,8)$, i.e. 8 electrons in 10 active orbitals, was used in combination with a $6-311 G^{*}$ basis for the rotational and $3-211 \mathrm{G}^{*}$ for vibrational calculations. The smaller basis for the vibrational calculations is precipitated by the large numbers of displacements necessary to integrate all vibrational degrees of freedom numerically. The frequency calculations required for determining the harmonic vibrational wavefunctions have been carried out by solving for the Hessian for all degrees of freedom using CAS-SCF(10,8)/6-311G* in Molpro. ${ }^{52}$ Integration over rotational and vibrational wavefunctions has been carried out numerically using quadrature, with the wavefunctions determined using the rigid rotor and harmonic approximations respectively. Finally, the elastic scattering has been calculated using the AIXRD code. ${ }^{42}$

\section{Results}

\subsection{Effect of basis size and comparison to IAM}

We begin by examining the dependence of the calculated scattering signal on the $a b$ initio basis set for non-relativistic ground state Hartree-Fock (HF) wavefunctions. We also include in the comparison the scattering signal calculated by the independent atom model (IAM) using tabulated atomic form factors. ${ }^{33}$ These are calculated from relativistic HF (RHF) wavefunctions, ${ }^{29,31,33}$ but the difference is negligible for the light elements under consideration here. In each case, we evaluate the absolute percent difference (relative error), $|\% \Delta \tilde{I}(q)|$, for rotationally averaged scattering,

$$
\% \Delta \tilde{I}(q)=100 \times \frac{\tilde{I}_{\text {method }}(q)-\tilde{I}_{\text {ref }}(q)}{\tilde{I}_{\text {ref }}(q)},
$$

with the reference, $\tilde{I}_{\text {ref }}(q)$, defined as scattering from the HF/augcc-pVQZ wavefunction. The trigonal planar $\mathrm{BF}_{3}$ and the trigonal pyramidal $\mathrm{NH}_{3}$ molecules are used for the comparison, with their geometry optimised at the HF/aug-cc-pVQZ level using Molpro ${ }^{52}$ $\left(R_{\mathrm{BF}}=2.444 a_{0}\right.$ and $\left.R_{\mathrm{NH}}=1.886 a_{0}\right)$. The absolute percent difference (relative error) is shown in Fig. 2 as a function of the momentum transfer $q$. Notably, the errors remain significant at large $q$, corresponding to high-resolution data, meaning that the resolution attainable from diffraction is adversely affected. It is also worth noting that the errors shown are rotationally averaged, meaning that for anisotropic samples such as molecular crystals or aligned gas-phase molecules the errors can be even greater for specific orientations of the vector $\mathbf{q}$.

A striking feature in Fig. 2 is the poor performance by HF/STO-3G. This becomes less surprising if one considers that STO-3G reproduces the tabulated atomic form factors used in the IAM calculation poorly, while all the other basis sets, including 6-31G, converge to the tabulated form factors. ${ }^{53}$ Essentially, the STO-3G wavefunctions are insufficiently accurate even for single 

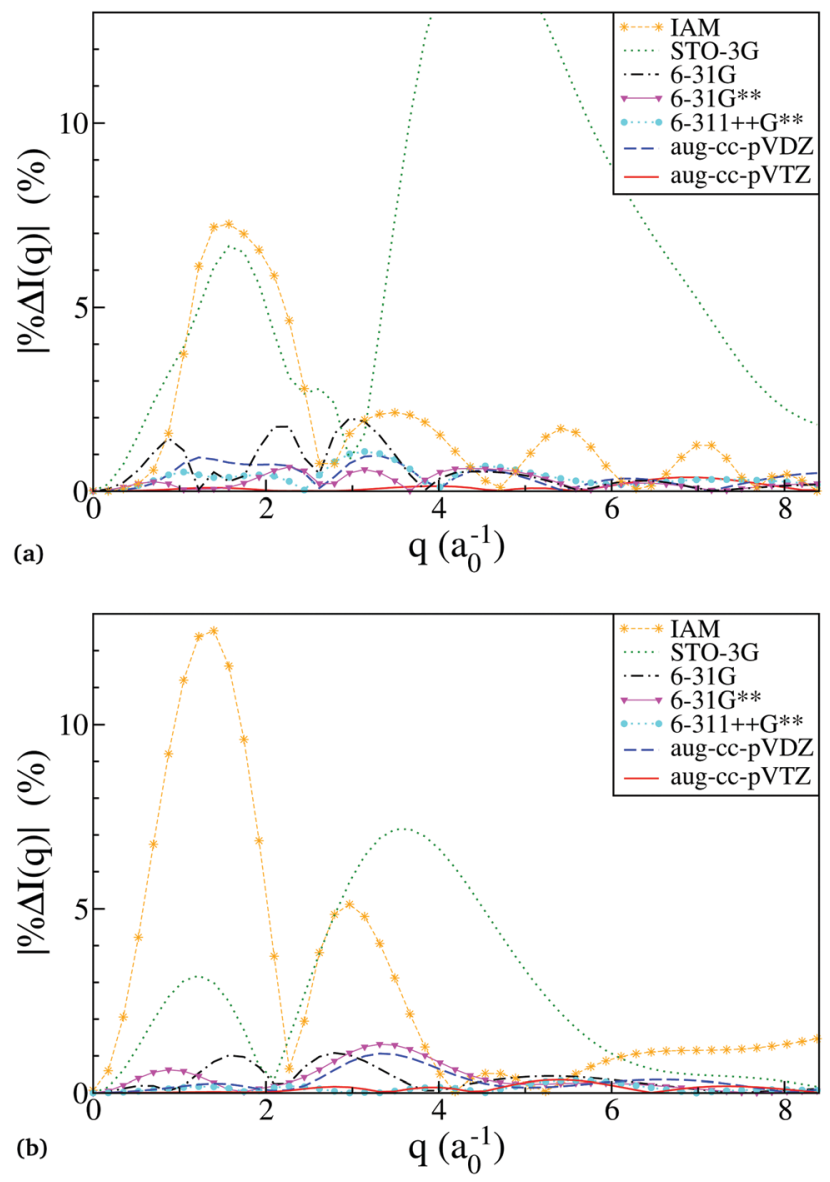

Fig. 2 The relative error in calculated scattering as a function of the basis used for the $a b$ initio electronic wavefunction, shown as percent error, eqn (9), for molecules (a) $\mathrm{BF}_{3}$, and (b) $\mathrm{NH}_{3}$. The nuclei are frozen at the HF/aug-cc-pVQZ ground-state energy-optimised geometry, and the scattering signal is rotationally averaged. (a) The relative error in scattering calculations for the molecule $\mathrm{BF}_{3}$. The truncated STO-3G curve peaks at $15 \%$ at around $q=4.4$. (b) The relative error in scattering calculations for the molecule $\mathrm{NH}_{3}$.

atoms. For all other basis sets, the discrepancy between the $a b$ initio scattering and the IAM relates to the failure of the latter to account for the redistribution of valence electrons due to chemical bonding. The smaller discrepancy between IAM and ab initio scattering in $\mathrm{BF}_{3}$ compared to $\mathrm{NH}_{3}$ brings to light that IAM performs better the larger the fraction of core electrons compared to valence electrons.

Table 1 shows the maximum and mean errors in the scattering, with the mean calculated as,

$$
\langle|\% \Delta \tilde{I}(q)|\rangle=\frac{1}{q_{\max }-q_{\min }} \int_{q_{\min }}^{q_{\max }}|\% \Delta \tilde{I}(q)| \mathrm{d} q,
$$

with the integration interval $\left[q_{\min }, q_{\max }\right]=[0,8.3] a_{0}{ }^{-1}$, while the error itself is calculated using eqn (9) above. The average ratio of maximum to mean for the HF calculations is 3.1, while the ratio for IAM is 4.3 , indicating that IAM is prone to greater systematic errors. The energy difference, $\Delta E=\left|E-E_{\text {ref }}\right|$, of each $a b$ initio calculation relative the HF/aug-cc-pVTZ reference is also provided in Table 1 . If we use $\Delta E$ as a proxy for the convergence of the
Table 1 Convergence and computational requirements for scattering from $\mathrm{HF}$ electronic wavefunctions calculated using various basis sets in molecules $\mathrm{BF}_{3}$ (top half) and $\mathrm{NH}_{3}$ (bottom half). Note that the ** and ++ basis sets are equivalent to * and + for molecules without hydrogen atoms, such as $\mathrm{BF}_{3}$. The $\Delta E$ for $\mathrm{NH}_{3}$ is given in units of $10^{-1} \times E_{\mathrm{h}}$

\begin{tabular}{|c|c|c|c|c|c|c|}
\hline \multirow[b]{2}{*}{ Method } & \multicolumn{2}{|c|}{ Error (\%) } & \multirow{2}{*}{$\frac{\Delta E}{\left(E_{\mathrm{h}}\right)}$} & \multirow{2}{*}{\multicolumn{2}{|c|}{$\frac{N_{\mathrm{g}} \quad N_{\mathrm{gp}}}{\left(\times 10^{3}\right)}$}} & \multirow[b]{2}{*}{ Speed up } \\
\hline & Mean & Max & & & & \\
\hline \multicolumn{7}{|l|}{$\mathrm{BF}_{3}$} \\
\hline IAM & 1.75 & 7.3 & - & - & & $41 \mathrm{k}$ \\
\hline STO-3G & 6.36 & 15 & 4.69 & 1.0 & 10 & 68 \\
\hline $6-31 \mathrm{G}$ & 0.54 & 2.0 & 0.27 & 1.4 & 21 & 31 \\
\hline $6-31 G^{* *}$ & 0.26 & 0.7 & 0.16 & 1.8 & 32 & 20 \\
\hline $6-311++\mathrm{G}^{* *}$ & 0.39 & 1.1 & 0.07 & 2.3 & 51 & 13 \\
\hline aug-cc-pVDZ & 0.41 & 1.0 & 0.13 & 4.3 & 105 & 6 \\
\hline aug-cc-pVTZ & 0.11 & 0.4 & 0.02 & 8.1 & 259 & 3 \\
\hline aug-cc-pVQZ & 0 & 0 & 0 & 14 & 655 & 1 \\
\hline \multicolumn{7}{|l|}{$\mathrm{NH}_{3}$} \\
\hline IAM & 2.94 & 13 & - & - & & $16 \mathrm{k}$ \\
\hline STO-3G & 2.54 & 7.2 & 7.73 & 0.1 & 1.5 & 173 \\
\hline $6-31 \mathrm{G}$ & 0.35 & 1.1 & 0.62 & 0.2 & 3 & 87 \\
\hline $6-31 G^{* *}$ & 0.38 & 1.3 & 0.29 & 0.2 & 6 & 43 \\
\hline $6-311++G^{* *}$ & 0.12 & 0.3 & 0.10 & 0.3 & 10 & 26 \\
\hline aug-cc-pVDZ & 0.31 & 1.1 & 0.20 & 0.6 & 19 & 14 \\
\hline aug-cc-pVTZ & 0.11 & 0.4 & 0.04 & 1.2 & 74 & 4 \\
\hline aug-cc-pVQZ & 0 & 0 & 0 & 2.2 & 260 & 1 \\
\hline
\end{tabular}

$a b$ initio calculations, we see a clear correlation between $\Delta E$ and the accuracy of the scattering. In $\mathrm{BF}_{3}$ it correctly identifies the best and the poorest performers, with a slight outperformance by the $6-31 \mathrm{G}^{* *}$ calculation. In $\mathrm{NH}_{3}, \Delta E$, correctly ranks the mean scattering error except for a swapping around of 6-31G and $6-31 \mathrm{G}^{* *}$, which rank sixth and fifth in terms of energy, but fifth and sixth in terms of mean scattering convergence.

\subsection{Scaling of AIXRD calculations}

The $a b$ initio scattering calculations essentially scale as the number of terms that have to be evaluated, which in turn depend on the size of the basis used to represent the electronic wavefunction. Table 1 shows the number of primitive Gaussian functions per calculation, $N_{\mathrm{g}}$, and more importantly, the number of non-zero unique Gaussian products, $N_{\mathrm{gp}}$. The computational effort to calculate the scattering scales linearly with the number of unique non-zero Gaussian products, $N_{\mathrm{gp}}$, rather than the actual number of Gaussian primitives, $N_{\mathrm{g}}$. For $\mathrm{BF}_{3}, N_{\mathrm{gp}} \approx N_{\mathrm{g}}{ }^{1.38}$, and for $\mathrm{NH}_{3} N_{\mathrm{gp}} \approx N_{\mathrm{g}}{ }^{1.58}$.

Table 1 also shows the speed-up, i.e. the time required for each scattering calculation relative the reference aug-cc-pVTZ wavefunction (the time for the $a b$ initio calculation itself is not included in the comparison). It is readily apparent that the IAM is several orders of magnitude faster than calculation of the scattering directly from the $a b$ initio wavefunction, and more than $10^{4}$ times faster than the reference calculation. However, this comparison verges on the meaningless, since IAM is based on tabulated values and thus does not require a scattering calculation at all, but merely interpolation and summation of tabulated values.

Pragmatically, it is worth noticing that in both molecules all the basis sets except STO-3G have a mean error of less than 
$0.6 \%$, showing that unless supreme accuracy is required, any medium-sized basis set is a reasonable choice. The $6-31 G^{* *}$ appears to lie in a sweet spot of low percent error and high computational efficiency, but the 6-31G basis set could also be used if an even larger speed-up is required. For higher accuracy, one would have to resort to post-HF methods (e.g. CASSCF or CASPT2) that better account for electron correlation. Examples where this becomes important include ground states with biradical character or excited electronic states (as in Section 4.3.4). Notably, post-HF methods follow the same scaling with basis size as discussed above, but with a larger prefactor. However, we emphasize that HF already delivers a significant improvement on IAM, at least for ground-state molecules containing light elements.

\subsection{Molecular scattering images}

The discussion in the following sections hinges around calculated X-ray scattering images that are 2D projections of the detector image presuming that the entire Ewald sphere is covered by the detector. The images are thus shown as polar plots in terms of the polar angle $(0 \leq \phi<2 \pi)$ and the radial angle $(0 \leq \theta \leq \pi)$ that specify the deflection of the scattered wavevector $\mathbf{k}$ relative the incident wavevector $\mathbf{k}_{0}$. The center of the image thus corresponds to $\theta=0$ (forward-scattering) and the outer rim to $\theta=\pi$ (backscattering). Since $q=2 k_{0} \sin \theta / 2$, we have that $q_{\max }=2 k_{0}$ at $\theta=\pi$. All images are shown without the outer absolute square in eqn (6) to make it easier to discern features at large values of $q$ in the figures. In several instances, difference images are shown to emphasize the changes in the scattering pattern upon excitation to a specific state. These difference images are calculated by subtracting a reference image from the excited state scattering image, with the subtraction done between images sans the absolute square. In the following, we first examine the effect of molecular symmetry on the scattering images (Section 4.3.1), and then move on to examine the different partial contributions to the scattering in the $\mathrm{CS}_{2}$ molecule (Sections 4.3.2-4.3.4).

4.3.1 Symmetry and centrosymmetry. The symmetry of a molecule relative the X-ray beam is reflected in the scattering image. For instance, if the molecule has a rotational symmetry axis parallel to the incoming $\mathrm{X}$-rays, this axis is replicated in the scattering. ${ }^{42}$ An interesting feature present in many of the scattering images shown here is centrosymmetry, i.e. the scattering signal for $(\phi, \theta)$ and $(\phi+\pi, \theta)$ is identical. A curious consequence of the centrosymmetry is that any odd-numbered rotational axis is doubled, as can be seen in the scattering patterns for $\mathrm{BF}_{3}$ $\left(D_{3 \mathrm{~h}}\right.$ point group) and the cyclopentadienyl anion, $\mathrm{C}_{5} \mathrm{H}_{5}{ }^{-}$ ( $D_{5 \mathrm{~h}}$ point group) in Fig. 3. The 3-fold axis for $\mathrm{BF}_{3}$ and the 5 -fold axis for $\mathrm{C}_{5} \mathrm{H}_{5}{ }^{-}$become 6- and 10-fold axes. In contrast, an even-numbered rotational axis, e.g. $C_{4}$, will result in the same rotational $C_{4}$ axis in the scattering image, with no apparent doubling since the images are already centrosymmetric. The mathematical background to this phenomenon is elaborated in the Appendix.

A necessary condition for the centrosymmetry is that the molecule contains a mirror plane orthogonal to the incoming $\mathrm{X}$-ray beam, as for instance is the case for the planar molecules in the two examples above. In contrast, Fig. 4 shows the scattering

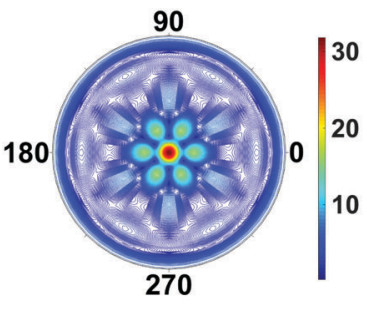

(a)

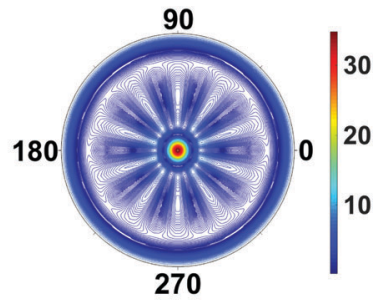

(b)
Fig. 3 Scattering images for (a) $\mathrm{BF}_{3}\left(D_{3 h}\right.$ point group) and (b) $\mathrm{C}_{5} \mathrm{H}_{5}{ }^{-}$ $\left(D_{5 \mathrm{~h}}\right.$ point group). The planar molecules are perpendicular to the incoming $X$-ray and the resulting diffraction image thus doubles the molecular rotational symmetry axis due to centrosymmetry. The value of $q_{\max }$ is $15.8 \AA^{-1}$.

image for $\mathrm{NF}_{3}$, a strongly-scattering ammonia analogue with $C_{3 \mathrm{v}}$ point group symmetry. The additional interference due to the out-of-plane nitrogen atom diminishes the centrosymmetry in the image, but the fundamental $C_{3}$ axis remains. Proceeding to a molecule with no discernable symmetry, 1,3 -cyclohexadiene $\left(C_{1}\right.$ point group), there is a corresponding absence of symmetry in the scattering image. Note, however, that some remnant of 'not-quite' centrosymmetry remains even in this image. This simply reflects the approximate degree of mirror symmetry perpendical to the incoming X-ray.

4.3.2 $\mathrm{CS}_{2}$ vibrations. We now examine the effect of vibrational states on the scattering pattern of $\mathrm{CS}_{2}$. The rotational states are not included at this stage, in order to keep the comparison as simple as possible. The modulations of the static scattering patterns will come from the changes in the distribution of relative atomic positions in the excited vibrational states, with scope from interferences due to the nodes in the vibrational wavefunctions. We consider the molecule $\mathrm{CS}_{2}$. In the ground state $\mathrm{CS}_{2}$ is linear with a C-S bond distance of $1.584 \AA$. The vibrational wavefunctions are calculated in the harmonic approximation. The normal mode frequencies are calculated at the $\operatorname{CAS}(10,8)$ SCF/6-311G* level of theory, and compare favourably with experimental values from the NIST database (see Table 2 ), with the differences less than $3 \%$.

The difference scattering images for $\mathrm{CS}_{2}$ shown in Fig. 5 are calculated from the full CAS-SCF electronic wavefunction and the vibrational wavefunctions. In each image, the vibrational wavefunction has one quantum of excitation in a different

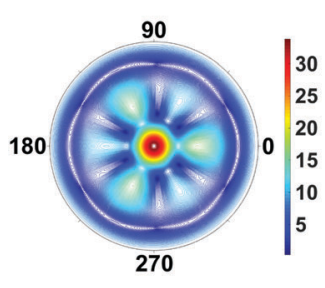

(a)

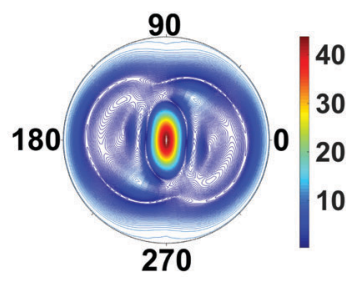

(b)
Fig. 4 Scattering images for (a) $\mathrm{NF}_{3}\left(C_{3 v}\right.$ point group) and (b) 1,3-cyclohexadiene $\left(C_{1}\right.$ point group). The main plane of the molecules is aligned perpendicular to the incoming $X$-ray. The absence of a mirror plane orthogonal to the incoming $X$-rays removes or diminishes centrosymmetry in the images. The value of $q_{\max }$ is $15.8 \AA^{-1}$. 
Table 2 Comparison between experimental and calculated frequencies for $\mathrm{CS}_{2}$ in the electronic ground state. The frequencies have been calculated using the analytical Hessian at the CAS(10,8)-SCF/6-311G* level of theory

\begin{tabular}{llll}
\hline Exp. $\left(\mathrm{cm}^{-1}\right)$ & Calc. $\left(\mathrm{cm}^{-1}\right)$ & $\Delta(\%)$ & Mode \\
\hline 1535 & 1491 & 2.9 & Symmetric \\
658 & 652 & 1.0 & Asymmetric $^{a}$ \\
397 & 399 & 0.6 & Bending $^{a}$
\end{tabular}

${ }^{a}$ Note that the bending mode is doubly degenerate.

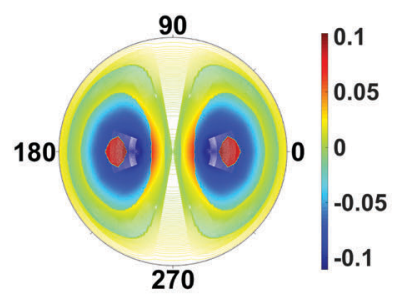

(a)

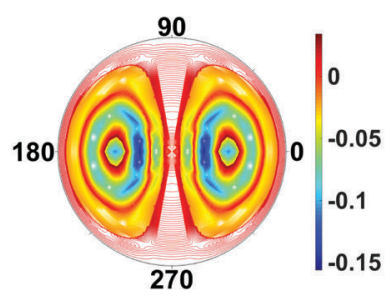

(b)

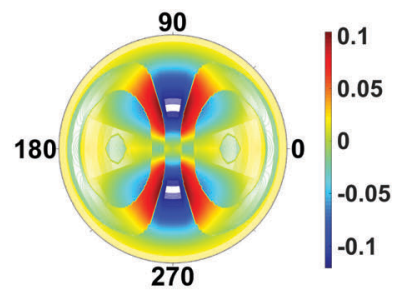

(c)

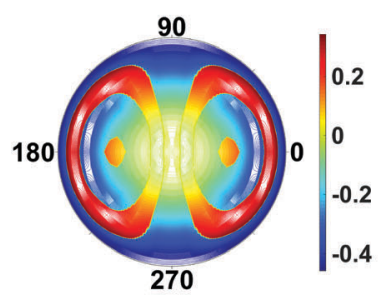

(d)
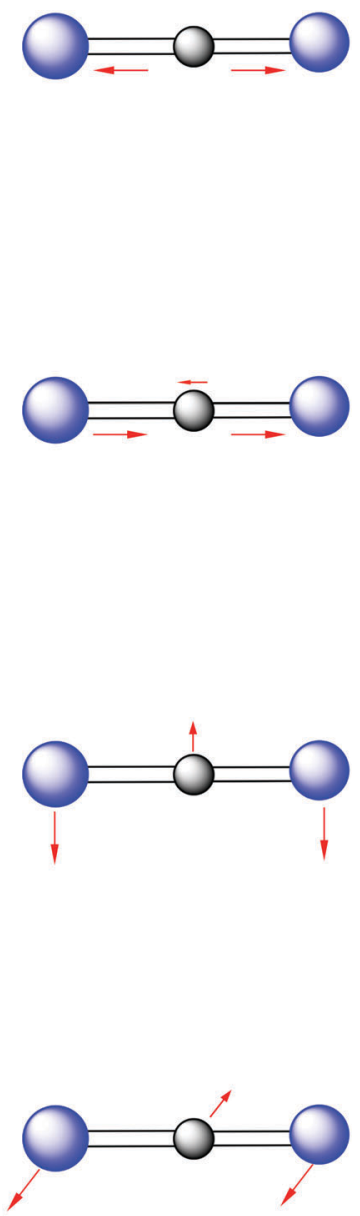

Fig. 5 Difference scattering images for each of the normal vibrational modes of $\mathrm{CS}_{2}$. The vibrational states are specified as $\left|\nu_{1} \nu_{2} \nu_{3} \nu_{4}\right\rangle_{\text {vib, }}$, with the order of vibrational quantum numbers corresponding to descending energy (see Table 2). The following vibrational states are considered: (a) symmetric stretch

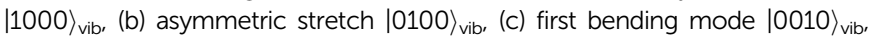
and (d) second bending mode $|0001\rangle_{\text {vib. }}$. The vibrational ground state $|0000\rangle_{\text {vib }}$ is taken as reference, and the value of $q_{\max }$ is $5.3 \AA^{-1}$ in each image. Note that the molecules are perfectly aligned with the vibration orthogonal to the direction of the incoming X-ray in (c) and parallel in (d).

vibrational mode. The symmetric, Fig. 5(a), and asymmetric, Fig. 5(b), stretches give rise to overall similar changes in the scattering pattern, but the asymmetric stretch has additional interferences rings due to the broken symmetry in the C-S bond distances. Likewise, the two bending modes generate very similar scattering patterns. The differences here are due to the bending mode in Fig. 5(c) being oriented perpendicular to the incoming X-ray (in-plane), while it is directed toward the incoming X-ray (out-of-plane) in Fig. 5(d). Whether these two modes can be distinguished is therefore dependent on the degree of orientation of the molecule. It is worth pointing out that since the $\mathrm{S}$ atoms are comparatively heavy, the vibrations in $\mathrm{CS}_{2}$ have small amplitudes. In terms of the asymmetric stretch and the bending modes, one can essentially consider these vibrations as small displacements of the central $\mathrm{C}$ atom relative two stationary $\mathrm{S}$ atoms. The changes in the scattering pattern due to vibrational state are therefore only on the order of $1 \%$ or less.

4.3.3 $\mathbf{C S}_{2}$ rotations. Next, we examine the effect of different rotational states calculated in the rigid rotor approximation on the scattering images. We continue to consider the $\mathrm{CS}_{2}$ molecule. Since this symmetric top molecule is linear, the $K$ quantum number is zero. Effectively, this reduces the rotational wavefunctions to spherical harmonics. The different values of $J$ and $M$ produce a various types of precession around the laboratory frame $z$-axis, leading to probability distributions that correspond to the spherical harmonics.

The calculated difference images are shown in Fig. 6. The first overall observation is that the effect of changes in rotational quantum state has a strong impact on the scattering image, with the signal changing more than an order of magnitude in specific scattering directions. Examining the individual images in Fig. 6, we see that the images fall into several categories. Fig. 6(a), (d), and (e), are quite similar. They correspond to scattering from dumb-bell or p-orbital type shapes of the rotational wavefunction, with Fig. 6(d) the most elongated in real space and Fig. 6(a) the least. The scattering images in Fig. 6(b), (c) and (f), on the other hand, correspond to doughnut-like shapes, with Fig. 6(f) the flattest in real space and Fig. 6(b) and (c) identical since the difference between them is a phase-factor in the wavefunction which does not affect the scattering. Looking at the overall effect of the rotational states on the scattering, it is greater than that of vibrations.

4.3.4 $\mathrm{CS}_{2}$ rotational, vibrational, and electronic states. We move on to simultaneously considering both rotational and vibrational states. Fig. 7 shows difference images for the $|101\rangle_{\text {rot }}|1111\rangle_{\text {vib }}$ and $|100\rangle_{\text {rot }}|1111\rangle_{\text {vib }}$ states. The trends observed when considering each type of motion separately, as in previous sections, are preserved. The rotational states lead to strong, order of magnitude, changes in specific scattering directions (translating into specific pixels on the detector), while the vibrational states leave much weaker signatures on the order of $<1 \%$, which is unsurprising given that only small harmonic oscillations around the equilibrium geometry are considered. If larger amplitude motion were included, the changes in molecular geometry would indeed leave a very strong signature in the scattering and eventually dominate all other contributions. 


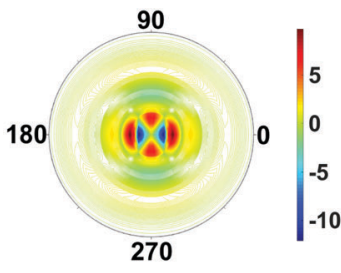

(a)

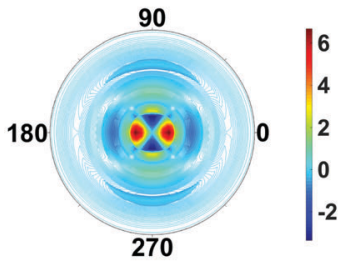

(c)

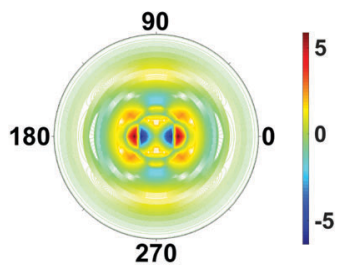

(e)

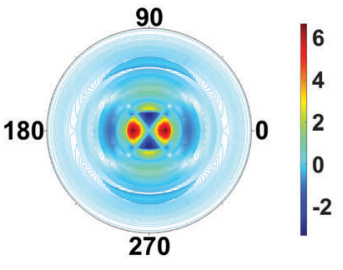

(b)

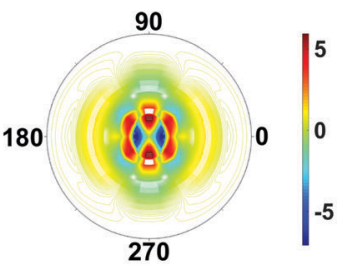

(d)

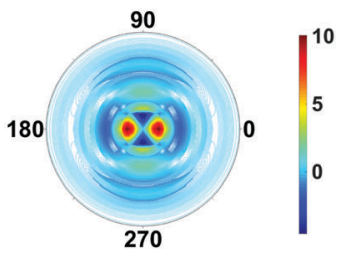

(f)
Fig. 6 Difference scattering images for rotational states in $\mathrm{CS}_{2}$, with the ground rotational state $|000\rangle_{\text {rot }}$ taken as reference. The following rotational states $|J K M\rangle_{\text {rot }}$ are considered: (a) $|100\rangle_{\text {rot' }}$ (b) $|101\rangle_{\text {rot' }}$ (c) $|10-1\rangle_{\text {rot, }}$ (d) $|200\rangle_{\text {rot }}$ (e) $|201\rangle_{\text {rot }}$ and (f) $|202\rangle_{\text {rot }}$. For the linear $\mathrm{CS}_{2}$ molecule, with $K=0$ by definition, the images essentially reflect the shape of the spherical harmonics, with each of the rotational states leaving a strong signature in the scattering. The value of $q_{\max }$ is $5.3 \AA^{-1}$ throughout.

As the total molecular wavefunction is discussed in this section, we also consider changes in the electronic state of the molecule. In Fig. 8(a) the changes in scattering pattern due to a vertical excitation from the $\mathrm{CS}_{2}$ ground $\mathrm{X}$ state to the bright excited B state are shown. The redistribution of the electrons in the molecule leads to a distinct change in the scattering pattern, not quite on the same order as the effect of rotations, but significantly stronger than the effect of small equilibrium vibrations. It is important to point out, however, that since the

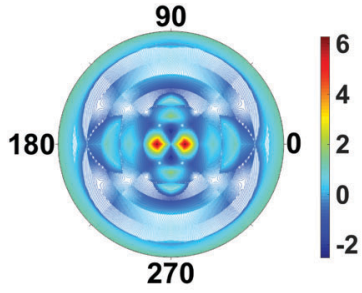

(a)

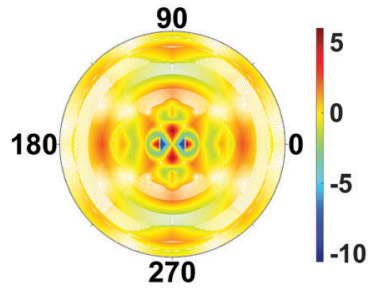

(b)
Fig. 7 Difference scattering images for $\mathrm{CS}_{2}$ in the electronic ground state for combined rotational-vibrational molecular states (a) $|101\rangle_{\text {rot }}|1111\rangle_{\text {vib, }}$ and (b) $|100\rangle_{\text {rot }}|1111\rangle_{\text {vib. }}$. The reference scattering image corresponds to the overall ground state $\left(|000\rangle_{\text {rot }}|0000\rangle_{\text {vib }}\right)$. The shape of the scattering pattern is similar in the two examples, but with inverted intensity. The value of $q_{\max }=5.3 \AA^{-1}$.

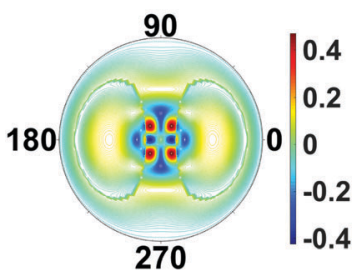

(a)

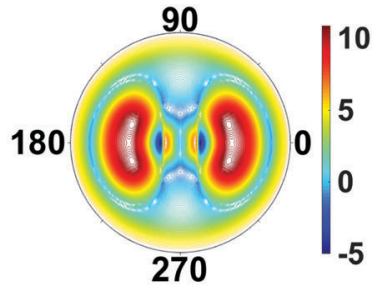

(b)
Fig. 8 Difference scattering images for $\mathrm{CS}_{2}$ in the optically bright excited electronic $|B\rangle$ state and the electronic ground state $|X\rangle$ in (a) the ground state geometry, i.e. vertical excitation, and (b) the B state equilibrium geometry. The image (b) emphasizes the effect of molecular geometry on the scattering images. The value of $q_{\max }=5.3 \AA^{-1}$, and the incoming $\mathrm{X}$-ray is perpendicular to the plane of the molecule in both cases.

equilibrium geometry of the electronic B state is different from the ground $\mathrm{X}$ state, nuclear motion necessarily ensues upon excitation, and these large-amplitude vibrations have a strong effect on the diffraction pattern. It is therefore non-trivial in general to separate the contributions from electronic redistribution and nuclear motion. As an illustration, Fig. 8(b) shows the scattering from the molecule in the electronic B state at the B-state equilibrium geometry, rather than the ground state geometry. The change in geometry gives a very large change in the scattering, and overwhelms the effect of rotation and equilibrium vibrations.

\section{Conclusions}

We have examined X-ray diffraction images from molecules in specific electronic, vibrational, and rotational states, and find that the scattering images carry distinct fingerprints of the total molecular wavefunction. The differences in intensity between different rotational states are an order of magnitude in specific directions of scattering (i.e. towards particular positions on the detector). In contrast, the changes due to different vibrational states are modest, about $1 \%$ or less. This is a consequence of the fact that low-lying vibrational states are considered, implying small and local nuclear displacements around equilibrium positions and hence no net change in molecular geometry. A technical point is that the integration over the multidimensional vibrational wavefunction is time-consuming, and given the small nuclear displacements considered in the harmonic approximation one could, in the future, examine the possibility of interpolating the electronic wavefunction coefficients. Electronic states also affect the scattering, ${ }^{42,54,55}$ with effects comparable to those of rotations in the presence of significant orientation or alignment. However, in most situations electronic excitation leads to changes in the nuclear geometry, ${ }^{56,57}$ since the potential energy surfaces associated with different electronic states are rarely parallel. This leads to very strong changes in the scattering, associated with the change in molecular geometry. An interesting point is that the greater the redistribution of electrons during vertical excitation of the molecule, and hence stronger signature of the electronic state, 
the greater is the likely effect on the nuclear motion, which itself bears an even stronger signature. Overall, it is apparent that disentangling different contributions, especially the vibrational and the electronic, to the scattering is not trivial.

The AIXRD calculations, which yield the elastic X-ray scattering directly from the $a b$ initio wavefunction, convincingly show the shortcomings of the simple but widely used independent atom model (IAM). The discrepancy between IAM and the more accurate AIXRD calculations persists even if full rotational averaging is considered. Although fitted generalized form factors can be used to address the deficiencies inherent in IAM, advances in computing and modern electronic structure codes make AIXRD calculations feasible, with large potential gains in accuracy and generality. It is particularly encouraging that the description of the scattering improves significantly already at quite modest levels of theory. This suggests that $a b$ initio $\mathrm{HF}$ calculations with reasonable basis sets or even density functional theory (DFT) could be used to determine molecular form factors for ground state molecules. A practical point is that the energy convergence of $a b$ initio electronic structure calculations serves as an adequate proxy for the quality of the wavefunction and thus the calculated scattering. Future work will examine if X-ray scattering may resolve more subtle aspects of fully coupled rovibronic states using higher-level spectroscopicaccuracy theory. ${ }^{58-60}$ Finally, given recent advances in ultrafast $\mathrm{X}$-ray scattering, ${ }^{4,61,62}$ the greatest value of the current study is that it demonstrates that AIXRD can be effectively combined with calculations that include nuclear degrees of freedom, thus opening the door for calculating the signals from ultrafast dynamics. ${ }^{4,61,63}$

\section{Appendix}

\section{Image symmetry and Friedel's law}

The centrosymmetry or near-centrosymmetry observed in many of the scattering images relates to the mirror symmetry of the molecule relative the direction of the incoming X-ray. Assuming that X-rays enter along the $z$-axis, $\mathbf{k}_{0}=\left(0,0, k_{0}\right)$, a pair of momentum transfer vectors corresponding to two points on the opposite sides of the detector are given by $\mathbf{q}=\left(q_{x}, q_{y}, q_{z}\right)$ and $\mathbf{q}^{\prime}=\left(-q_{x},-q_{y}, q_{z}\right)$. Centrosymmetry requires that $\left|F\left(\mathbf{q}^{\prime}\right)\right|^{2}=|F(\mathbf{q})|^{2}$.

If we express the electron density as a sum over Gaussian functions, each a product of $x, y$, and $z$ components, ${ }^{42}$

$$
\rho(x, y, z)=\sum_{i} c_{i} g_{i}(x) g_{i}(y) g_{i}(z)
$$

the Fourier transform of the density for the $\mathbf{q}^{\prime}$ vector becomes,

$$
F\left(\mathbf{q}^{\prime}\right)=\sum_{i} F_{x}\left[g_{i}(x)\right]\left(-q_{x}\right) F_{y}\left[g_{i}(y)\right]\left(-q_{y}\right) F_{z}\left[g_{z}(z)\right]\left(q_{z}\right) .
$$

Friedel's law for Fourier transforms, $F(q)=F^{*}(-q)$, is valid for real-valued functions such as the electron density. It applies to the $x$ and $y$ components in eqn (12), but not the $z$-component. However, if the overall electron density is symmetric with regards to the $z$-axis, the net Fourier transform along the $z$-axis must be real-valued (in practice this occurs by collecting symmetry-related pairs in the sum in eqn (12) with identical $x$ and $y$ components and complex conjugate $z$ components). In that situation the Fourier transform of the $z$-component does not invalidate Friedel's law for the overall expression and we obtain $F^{*}\left(\mathbf{q}^{\prime}\right)=F(\mathbf{q})$, which in turn implies $\left|F\left(\mathbf{q}^{\prime}\right)\right|^{2}=|F(\mathbf{q})|^{2}$ as required for centrosymmetry.

It is worth noting that Friedel's law always applies to the $x$ and $y$ components (since the electron density is real), and that the degree of centrosymmetry only relates to the degree of symmetry along the $z$-axis, with strict centrosymmetry requiring a mirror plane orthogonal to the incoming X-ray. In fact, a quicker and more elegant way to arrive at this result is to consider that the mirror symmetry operation, when present in the molecule, can be applied to the $\mathbf{q}^{\prime}$ vector such that $\left(-q_{x},-q_{y}, q_{z}\right) \rightarrow\left(-q_{x},-q_{y},-q_{z}\right)$, at which point the three-dimensional version of Friedel's law, $F(\mathbf{q})=F^{*}(-\mathbf{q})$, applies.

\section{Rotational matrix}

Rotations correspond to changes in the frame (coordinate system) given by the Euler angles $(\alpha, \beta, \gamma)$ show in Fig. 9. Coordinates in $(\xi, \eta, \zeta)$ axis systems can be related to the $(x, y, z)$ system by the standard rotation matrix (see eqn (10-5) and (10-7) in ref. 64 or Table I-1 in ref. 65),

$$
\left(\begin{array}{c}
x_{i} \\
y_{i} \\
z_{i}
\end{array}\right)=\left(\begin{array}{ccc}
\lambda_{x \xi} & \lambda_{x \eta} & \lambda_{x \zeta} \\
\lambda_{y \xi} & \lambda_{y \eta} & \lambda_{y \zeta} \\
\lambda_{z \xi} & \lambda_{z \eta} & \lambda_{z \zeta}
\end{array}\right)\left(\begin{array}{c}
\xi_{i} \\
\eta_{i} \\
\zeta_{i}
\end{array}\right),
$$

with the column vectors of the rotation matrix $\lambda$ defined as,

$$
\begin{aligned}
& \lambda_{i 1}=\left(\begin{array}{l}
\cos \alpha \cos \beta \cos \gamma-\sin \beta \sin \gamma \\
-\cos \alpha \cos \beta \sin \gamma-\sin \beta \cos \gamma \\
\sin \alpha \cos \beta
\end{array}\right) \\
& \lambda_{i 2}=\left(\begin{array}{l}
\cos \alpha \sin \beta \cos \gamma+\cos \beta \sin \gamma \\
-\cos \alpha \sin \beta \sin \gamma+\cos \beta \cos \gamma \\
\sin \alpha \sin \beta
\end{array}\right) \\
& \lambda_{i 3}=\left(\begin{array}{l}
-\sin \alpha \cos \gamma \\
\sin \alpha \sin \gamma \\
\cos \alpha
\end{array}\right),
\end{aligned}
$$

where $(\alpha, \beta, \gamma)$ are the Euler angles specified in Fig. 9. Since the rotation matrix $\lambda$ is unitary, inverse rotations are given by the transpose of the matrix.

\section{Classification of rigid rotor wavefunctions}

The general form of this mathematical expression can be obtained calculating the rotational Hamiltonian,

$$
\hat{H}_{\text {rot }}=\hbar^{2}\left(A_{\mathrm{e}} J_{\mathrm{a}}^{2}+B_{\mathrm{e}} J_{\mathrm{b}}{ }^{2}+C_{\mathrm{e}} J_{\mathrm{c}}{ }^{2}\right),
$$

where $A_{\mathrm{e}}, B_{\mathrm{e}}$ and $C_{\mathrm{e}}$ are the rotational constants of the molecule and $J_{\alpha}$ are the principal inertial axes of the equilibrium configuration. The classification of molecules according to their moments of inertia 


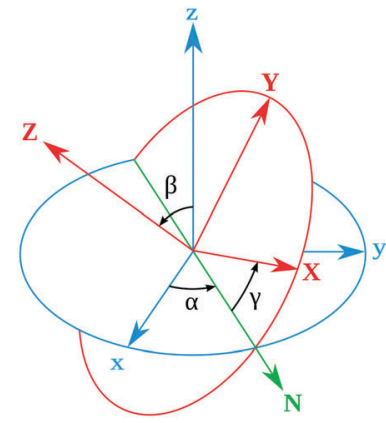

Fig. 9 Definition of the rotational Euler angles $(\alpha, \beta, \gamma)$. The two frames are related by the rotation matrix (see Appendix).

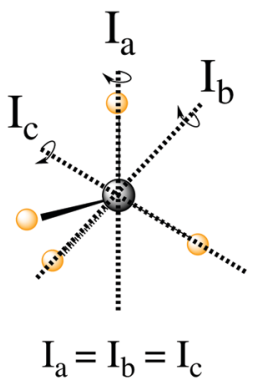

Spherical Top

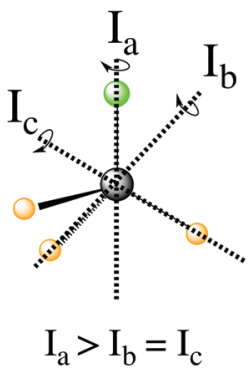

Symmetric Top

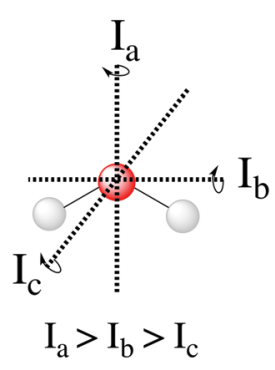

Asymmetric Top
Fig. 10 Representation of the different types of molecules depending on their rotational symmetry. The relative size of the different moments of inertia, $I_{n}$, determines the rotational symmetry of a molecule. The spherical tops are characterized by $I_{\mathrm{a}}=I_{\mathrm{b}}=I_{\mathrm{c}}$, the symmetric tops by two identical moments of inertia, subdivided into oblate symmetric tops (disc-shaped), $I_{\mathrm{a}}=I_{\mathrm{b}}>I_{\mathrm{c}}$, and prolate symmetric tops (cylindrical), $I_{\mathrm{a}}>I_{\mathrm{b}}=I_{\mathrm{c}}$, and finally asymmetric tops have $I_{\mathrm{a}}>I_{\mathrm{b}}>I_{\mathrm{c}}$.

is given in Fig. 10. Depending on the moments of inertia around the three axes of rotation, the molecules are classified as either spherical, symmetric and asymmetric tops. Each of these classes behaves differently under rotation and thus have different rotational wavefunctions.

\section{Rotational wavefunctions for spherical and symmetric tops}

These two classes of molecules share the same wavefunctions to describe their rotational states and can be expressed as a combination of spherical harmonics multiplied by a phase. The Schrödinger equation for a prolate symmetric top is,

$$
\hbar^{-2}\left[A_{\mathrm{e}} J_{\mathrm{a}}{ }^{2}+B_{\mathrm{e}}\left(J_{\mathrm{b}}{ }^{2}+J_{\mathrm{c}}{ }^{2}\right)\right] \Phi_{\mathrm{rot}}(\theta, \phi, \chi)=E_{\mathrm{rot}} \Phi_{\mathrm{rot}}(\theta, \phi, \chi) .
$$

The angular momentum operators $\hat{J}^{2}, \hat{J}_{\gamma}$, and $\hat{J}_{z}$ commute with each other and their eigenfunctions are the so-called rotation matrices. If we select the principal axis of rotation as $z$ (known as the $I_{\mathrm{r}}$ convention), we can rewrite the equation in terms of angular momentum operators,

$$
\hbar^{2}\left[B_{\mathrm{e}} \hat{J}^{2}+\left(A_{\mathrm{e}}-B_{\mathrm{e}}\right) \hat{J}_{z}^{2}\right] \Phi_{\mathrm{rot}}(\theta, \phi, \chi)=E_{\mathrm{rot}} \Phi_{\mathrm{rot}}(\theta, \phi, \chi) .
$$

Using the rotation matrices and eigenvalues of the operators we can obtain the representation of the wavefunction for a prolate symmetric top,

$$
\begin{aligned}
\Phi_{\text {rot }}(\theta, \phi, \chi) & =\left[(2 J+1) /\left(8 \pi^{2}\right)\right]^{1 / 2}\left[D_{M K}^{(J)}(\theta, \phi, \chi)\right]^{*} \\
& =(-1)^{M-K}\left[(2 J+1) /\left(8 \pi^{2}\right)\right]^{1 / 2}\left[D_{-M-K}^{(J)}(\theta, \phi, \chi)\right],
\end{aligned}
$$

where $D_{M K}^{(J)}$ are the rotation matrices and $J, K$ and $M$ the rotational quantum numbers, with $|K|$ and $|M|$ having allowed values $\leq J$. The equation can be rewritten as a function of the Euler angles $(\theta, \phi, \chi)$,

$$
\begin{aligned}
& \Phi(\theta, \phi, \chi)=X_{K M}^{J} \mathrm{e}^{i m \phi} \mathrm{e}^{i k \chi} \\
& \times\left[\sum_{\sigma}(-1)^{\sigma} \frac{\cos \left(\frac{\theta}{2}\right)^{2 J+K-M-2 \sigma}-\sin \left(\frac{\theta}{2}\right)^{M-K+2 \sigma}}{\sigma !(J-M-\sigma) !(M-K+\sigma) !(J-K-\sigma) !}\right]
\end{aligned}
$$

where $X_{K M}^{J}$ is a normalization constant with the form,

$$
X_{K M}^{J}=\left[(J+M) !(J-M) !(J+K) !(J-K) !(2 J+1) /\left(8 \pi^{2}\right)\right]^{1 / 2} .
$$

It is important to note that this wavefunction is only valid if our molecule has its origin of coordinates in the center of mass and the principal axis of symmetry is oriented along the $z$-axis. Other conventions can be used and they depend on the orientation of the coordinate system and the molecular symmetry $\left(I I I^{\mathrm{r}}\right)$.

The Hamiltonian form will change depending on the type of molecule we choose but the wavefunction will be the same as for prolate symmetric top if the system has at least two axis of inertia with the same value (spherical, prolate and oblate symmetric top molecules).

\section{Rotational wavefunctions for asymmetric top molecules}

Asymmetric top molecules, such as water, are the most common. In this class, all three axes of inertia are different. To evaluate the wavefunction of an asymmetric molecule we need a linear combination of symmetric top wavefunctions $(|J K M\rangle)$,

$$
\Phi(\theta, \phi, \chi)=a|J K M\rangle+b\left|J^{\prime} K^{\prime} M^{\prime}\right\rangle .
$$

To obtain these basis and their coefficients we need to diagonalize the rotational Hamiltonian expressed in the $|J K M\rangle$ basis,

$$
\begin{aligned}
\hat{H}_{\text {rot }}= & \hbar^{-2}\left[\left[\left(B_{\mathrm{e}}+C_{\mathrm{e}}\right) / 2\right] \hat{J}^{2}+\left[A_{\mathrm{e}}-\left(B_{\mathrm{e}}+C_{\mathrm{e}}\right) / 2\right] \hat{J}_{z}^{2}\right. \\
& \left.+\left[\left(B_{\mathrm{e}}-C_{\mathrm{e}}\right) / 4\right]\left[\left(\hat{J}_{M}^{+}\right)^{2}+\left(\hat{J}_{M}^{-}\right)^{2}\right]\right] .
\end{aligned}
$$

For each $J$ we will have $(2 J+1)$ states in $K$ and $M$, the basis need to be build as $\pm K$ linear combinations to be eigenfunctions of the operators presented in $H_{\text {rot }}$. We can classify them by the symbols $O^{+}, O^{-}, E^{+}$and $E^{-}$depending on the value of $K$ (even or odd) and the sign of the linear combination (+ or - ). The resultant basis functions can be directly diagonal elements of the Hamiltonian and therefore eigenvectors of it. In case we have off-diagonal elements we need to proceed with the diagonalisation of the system, obtaining linear combination of basis as rotational wavefunctions (see Table 3). 
Table 3 Asymmetric top wavefunctions for the three first $J$ values and all $K$. The nomenclature excludes $M$, since the equations are independent of $M . E, O,+$ and - depend on whether $K$ is odd or even and the positive or negative linear combination of $|J, K, M\rangle$. The $J=0$ and $J=1$ cases are nearly trivial, but calculation of $J=2$ requires diagonalization of the rotational Hamiltonian matrix

\begin{tabular}{ll}
\hline$J$ & Wavefunction \\
\hline 0 & $E^{+}=\left(\frac{1}{8 \pi^{2}}\right)^{1 / 2}$ \\
& $\left|1,1, O^{+}\right\rangle=[|1,1\rangle+|1,-1\rangle] / \sqrt{2}$ \\
1 & $\left|1,1, O^{-}\right\rangle=[|1,1\rangle-|1,-1\rangle] / \sqrt{2}$ \\
& $\left|1,0, E^{+}\right\rangle=|1,0\rangle$ \\
& $\left|2,2, E^{-}\right\rangle=[|2,2\rangle-|2,-2\rangle] / \sqrt{2}$ \\
& $\left|2,1, O^{+}\right\rangle=[|2,1\rangle+|2,-1\rangle] / \sqrt{2}$ \\
& $\left|2,1, O^{-}\right\rangle=[|2,1\rangle-|2,-1\rangle] / \sqrt{2}$ \\
& $\Phi_{\text {rot }}^{+}\left(2,0, E^{+}\right)=\left[c^{-}|2,0\rangle+c^{+}|2,2\rangle\right] / \sqrt{2}$ \\
& $\Phi_{\text {rot }}^{-}\left(2,0, E^{+}\right)=\left[c^{-}|2,0\rangle-c^{+}|2,2\rangle\right] / \sqrt{2}$ \\
& \\
& \\
&
\end{tabular}

\section{Acknowledgements}

AK and AMC acknowledge funding from the European Union (FP7-PEOPLE-2013-CIG-NEWLIGHT) and the hospitality of Prof. Roland Lindh (Uppsala University). AK further acknowledges the Leverhulme Trust (RPG-2013-365) and sabbatical support from Magnus Bergvalls Stiftelse (Sweden).

\section{References}

1 M. Dell'Angela, T. Anniyev, M. Beye, R. Coffee, A. Fohlisch, J. Gladh, T. Katayama, S. Kaya, O. Krupin, J. LaRue, A. Mogelhoj, D. Nordlund, J. K. Norskov, H. Oberg, H. Ogasawara, H. Ostrom, L. G. M. Pettersson, W. F. Schlotter, J. A. Sellberg, F. Sorgenfrei, J. J. Turner, M. Wolf, W. Wurth and A. Nilsson, Science, 2013, 339, 1302.

2 M. Beye, H. Öberg, H. Xin, G. L. Dakovski, M. Dell'Angela, A. Föhlisch, J. Gladh, M. Hantschmann, F. Hieke, S. Kaya, D. Kühn, J. LaRue, G. Mercurio, M. P. Minitti, A. Mitra, S. P. Moeller, M. L. Ng, A. Nilsson, D. Nordlund, J. Norskov, H. Öström, H. Ogasawara, M. Persson, W. F. Schlotter, J. A. Sellberg, M. Wolf, F. Abild-Pedersen, L. G. M. Pettersson and W. Wurth, J. Phys. Chem. Lett., 2016, 7, 3647-3651.

3 V. S. Petrović, M. Siano, J. L. White, N. Berrah, C. Bostedt, J. D. Bozek, D. Broege, M. Chalfin, R. N. Coffee, J. Cryan, L. Fang, J. P. Farrell, L. J. Frasinski, J. M. Glownia, M. Gühr, M. Hoener, D. M. P. Holland, J. Kim, J. P. Marangos, T. Martinez, B. K. McFarland, R. S. Minns, S. Miyabe, S. Schorb, R. J. Sension, L. S. Spector, R. Squibb, H. Tao, J. G. Underwood and P. H. Bucksbaum, Phys. Rev. Lett., 2012, 108, 253006.

4 M. P. Minitti, J. M. Budarz, A. Kirrander, J. S. Robinson, D. Ratner, T. J. Lane, D. Zhu, J. M. Glownia, M. Kozina, H. T. Lemke, M. Sikorski, Y. Feng, S. Nelson, K. Saita,
B. Stankus, T. Northey, J. B. Hastings and P. M. Weber, Phys. Rev. Lett., 2015, 114, 255501.

5 K. H. Kim, J. G. Kim, S. Nozawa, T. Sato, K. Y. Oang, T. W. Kim, H. Ki, J. Jo, S. Park, C. Song, T. Sato, K. Ogawa, T. Togashi, K. Tono, M. Yabashi, T. Ishikawa, J. Kim, R. Ryoo, J. Kim, H. Ihee and S. i. Adachi, Nature, 2015, 518, 385.

6 R. Neutze, R. Wouts, D. van der Spoel, E. Weckert and J. Hajdu, Nature, 2000, 406, 752.

7 J. R. Helliwell, Science, 2013, 339, 146.

8 E. F. Garman, Science, 2014, 343, 1102.

9 C. S. Barrett, Phys. Rev., 1928, 32, 22.

10 P. Debye, L. Bewilogua and F. Ehrhardt, Phys. Z., 1929, 30, 84. 11 E. O. Wollan, Phys. Rev., 1931, 37, 862.

12 M. H. Pirenne, The Diffraction of X-Rays and Electrons by Free Molecules, Cambridge University Press, London, 1946.

13 D. R. Chipman and L. D. Jennings, Phys. Rev., 1963, 132, 728. 14 M. P. Minitti, J. M. Budarz, A. Kirrander, J. Robinson, T. J. Lane, D. Ratner, K. Saita, T. Northey, B. Stankus, V. Cofer-Shabica, J. Hastings and P. M. Weber, Faraday Discuss., 2014, 171, 81.

15 J. M. Budarz, M. P. Minitti, D. V. Cofer-Shabica, B. Stankus, A. Kirrander, J. B. Hastings and P. M. Weber, J. Phys. B, 2016, 49, 034001.

16 B. Stankus, J. M. Budarz, A. Kirrander, D. Rogers, J. Robinson, T. J. Lane, D. Ratner, J. Hastings, M. P. Minitti and P. M. Weber, Faraday Discuss., 2016, 194, 525-536.

17 J. Küpper, S. Stern, L. Holmegaard, F. Filsinger, A. Rouzée, A. Rudenko, P. Johnsson, A. V. Martin, M. Adolph, A. Aquila, S. Bajt, A. Barty, C. Bostedt, J. Bozek, C. Caleman, R. Coffee, N. Coppola, T. Delmas, S. Epp, B. Erk, L. Foucar, T. Gorkhover, L. Gumprecht, A. Hartmann, R. Hartmann, G. Hauser, P. Holl, A. Hömke, N. Kimmel, F. Krasniqi, K.-U. Kühnel, J. Maurer, M. Messerschmidt, R. Moshammer, C. Reich, B. Rudek, R. Santra, I. Schlichting, C. Schmidt, S. Schorb, J. Schulz, H. Soltau, J. C. H. Spence, D. Starodub, L. Strüder, J. Thøgersen, M. J. J. Vrakking, G. Weidenspointner, T. A. White, C. Wunderer, G. Meijer, J. Ullrich, H. Stapelfeldt, D. Rolles and H. N. Chapman, Phys. Rev. Lett., 2014, 112, 083002. 18 B. Xie, L. Zhu, K. Yang, B. Zhou, N. Hiraoka, Y. Cai, Y. Yao, C. Wu, E. Wang and D. Feng, Phys. Rev. A: At., Mol., Opt. Phys., 2010, 82, 032501.

19 Y.-W. Liu, X.-X. Mei, X. Kang, K. Yang, W.-Q. Xu, Y.-G. Peng, N. Hiraoka, K.-D. Tsuei, P.-F. Zhang and L.-F. Zhu, Phys. Rev. A: At., Mol., Opt. Phys., 2014, 89, 014502.

20 L. Zhu, L. Wang, B. Xie, K. Yang, N. Hiraoka, Y. Cai and D. Feng, J. Phys. B, 2011, 44, 025203.

21 Y.-G. Peng, X. Kang, K. Yang, X.-L. Zhao, Y.-W. Liu, X.-X. Mei, W.-Q. Xu, N. Hiraoka, K.-D. Tsuei and L.-F. Zhu, Phys. Rev. A: At., Mol., Opt. Phys., 2014, 89, 032512.

22 C. Vallance, Phys. Chem. Chem. Phys., 2011, 13, 14427.

23 G. Sitz and R. L. Farrow, J. Chem. Phys., 1994, 101, 4682-4687. 24 A. D. Rudert, J. Martin, W.-B. Gao, J. B. Halpern and H. Zacharias, J. Chem. Phys., 1999, 111, 9549.

25 N. C. M. Bartlett, D. J. Miller, R. N. Zare, D. Sofikitis, P. T. Rakitzis and A. J. Alexander, J. Chem. Phys., 2008, 129, 084312.

26 N. Mukherjee and R. N. Zare, J. Chem. Phys., 2011, 135, 024201. 
27 N. Mukherjee, W. Dong, J. Harrison and R. Zare, J. Chem. Phys., 2013, 138, 051101.

28 P. Debye, Ann. Phys., 1915, 46, 809.

29 P. A. Doyle and P. S. Turner, Acta Crystallogr., Sect. A: Cryst. Phys., Diffr., Theor. Gen. Crystallogr., 1968, 24, 390-397.

30 J. H. Hubbell, W. J. Veigele, E. A. Briggs, R. T. Brown, D. T. Cromer and R. J. Howerton, J. Phys. Chem. Ref. Data, 1975, 4, 471.

31 A. G. Fox, M. A. O'Keefe and M. A. Tabbernor, Acta Crystallogr., Sect. A: Found. Crystallogr., 1989, 45, 786-793.

32 D. McMorrow and J. Als-Nielsen, Elements of Modern X-Ray Physics, Wiley-Blackwell, 2nd edn, 2011.

33 International Tables for Crystallography Volume C: Mathematical, Physical and Chemical Tables, ed. E. Prince, Wiley, 3rd edn, 2006.

34 A. O. Dohn, E. Biasin, K. Haldrup, M. M. Nielsen, N. E. Henriksen and K. B. Møller, J. Phys. B, 2015, 48, 244010.

35 R. F. Stewart, J. Bentley and B. Goodman, J. Chem. Phys., 1975, 63, 3786 .

36 J. Bentley and R. F. Stewart, J. Chem. Phys., 1975, 63, 3794.

37 R. F. Stewart, E. R. Davidson and W. T. Simpson, J. Chem. Phys., 1965, 42, 3175.

38 J. Epstein and R. F. Stewart, J. Chem. Phys., 1977, 66, 4057. 39 A. Debnarova and S. Techert, J. Chem. Phys., 2006, 125, 224101.

40 A. Debnarova, S. Techert and S. Schmatz, J. Chem. Phys., 2010, 133, 124309.

41 A. Debnarova, S. Techert and S. Schmatz, J. Chem. Phys., 2011, 134, 054302.

42 T. Northey, N. Zotev and A. Kirrander, J. Chem. Theory Comput., 2014, 10, 4911.

43 J. Yang, J. Beck, C. J. Uiterwaal and M. Centurion, Nat. Commun., 2015, 6, 8172.

44 K. Wang, V. McKoy, P. Hockett and M. S. Schuurman, Phys. Rev. Lett., 2014, 112, 113007.

45 P. Hockett, C. Z. Bisgaard, O. J. Clarkin and A. Stolow, Nat. Phys., 2011, 7, 612-615.

46 T. Fuji, Y.-I. Suzuki, T. Horio and T. Suzuki, Chem. - Asian J., 2011, 6, 3028-3034.
47 C. Z. Bisgaard, O. J. Clarkin, G. Wu, A. M. D. Lee, O. Gessner, C. C. Hayden and A. Stolow, Science, 2009, 323, 1464-1468.

48 D. Townsend, H. Satzger, T. Ejdrup, A. M. D. Lee, H. Stapelfeldt and A. Stolow, J. Chem. Phys., 2006, 125, 234302.

49 W. Schülke, Electron Dynamics by Inelastic X-Ray Scattering, Oxford Science Publications, 1st edn, 2007.

50 J. Bentley and R. F. Stewart, J. Comput. Phys., 1973, 11, 127.

51 I. Hargittai and M. Hargittai, Stereochemical applications of gas-phase electron diffraction: part $A$ the electron diffraction technique, VCH, 1st edn, 1988.

52 H.-J. Werner, P. J. Knowles, G. Knizia, F. R. Manby and M. Schütz, et al., MOLPRO, version 2012.1, a package of $a b$ initio programs, 2012.

53 T. Northey, PhD thesis, The University of Edinburgh, 2016. 54 A. Kirrander, J. Chem. Phys., 2012, 137, 154310.

55 H. J. Suominen and A. Kirrander, Phys. Rev. Lett., 2014, 112, 043002.

56 M. R. Pressprich, M. A. White and P. Coppens, J. Am. Chem. Soc., 1993, 115, 6444.

57 M. R. Pressprich, M. A. White, Y. Vekhter and P. Coppens, J. Am. Chem. Soc., 1994, 116, 5233.

58 A. Kirrander, C. Jungen and H. H. Fielding, Phys. Chem. Chem. Phys., 2010, 12, 8948.

59 A. Kirrander, J. Chem. Phys., 2010, 133, 121103.

60 A. Kirrander and C. Jungen, Phys. Rev. A: At., Mol., Opt. Phys., 2011, 84, 052512.

61 A. Kirrander, K. Saita and D. V. Shalashilin, J. Chem. Theory Comput., 2016, 12, 957-967.

62 C. C. Pemberton, Y. Zhang, K. Saita, A. Kirrander and P. M. Weber, J. Phys. Chem. A, 2015, 119, 8832.

63 R. Neutze, R. Wouts, S. Techert, J. Davidsson, M. Kocsis, A. Kirrander, F. Schotte and M. Wulff, Phys. Rev. Lett., 2001, 87, 195508.

64 P. R. Bunker and P. Jensen, Molecular Symmetry and Spectroscopy, NRC Research Press, 2nd edn, 1998.

65 J. E. Bright Wilson, J. C. Decius and P. C. Cross, Molecular Vibrations: The Theory of Infrared and Raman Vibrational Spectra, Dover, 1st edn, 1955. 